
\title{
BMJ Quality
}

\section{Improving Bisphosphonate Infusion Monitoring at Haematology Medical Day Unit}

\author{
Michal Wen Sheue Ong, Lydia Jones
}

To cite: Ong MWS, Jones L. Improving Bisphosphonate Infusion Monitoring at Haematology Medical Day Unit. BMJ Quality Improvement Reports 2017;6:u206586.w4692. doi:10.1136/bmjquality. u206586.w4692

Received 8 August 2016 Revised 25 October 2016

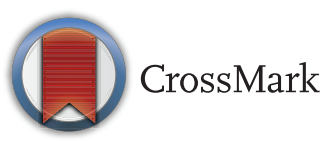

Epsom General Hospital, United Kingdom

Correspondence to Michal Wen Sheue Ong michalong@gmail.com

\section{ABSTRACT}

This project was started after an incident of bisphosphonate-induced hypocalcaemia in September 2015. As part of management of lytic bone lesions in patients with multiple myeloma were given either Zoledronic Acid or Pamidronate Disodium at our Haematology Day Unit. According to the British National Formulary (BNF), it is necessary to correct disturbances of calcium metabolism (e.g. vitamin D deficiency, hypocalcaemia) and consider dental checkups before starting bisphosphonate infusion due to the risk of osteonecrosis of the jaw. There was no formal checklist in place for all patients prior to starting bisphosphonate infusion. The aim of this quality improvement project was (1) to avoid preventable bisphosphonate induced adverse effects, (2) to improve safety of bisphosphonate prescribing and administration and (3) to increase patient's awareness of needing regular dental checks. Interventions were modified over multiple Plan-Do-Study-Act (PDSA) improvement cycles to improve bisphosphonate infusion monitoring and patient safety.There was an overall improvement in ensuring safety checks were done prior to administration of bisphosphonate infusion compared to baseline measurements. At baseline, $36 \%(n=9)$ of patients had a dental check within the last 6 months; after PDSA cycle 3, there was an improvement of up to $69 \%(n=11)$. All patients had renal function and bone profile checked prior to infusion from throughout the study. It was all recorded in the blood results section of the checklist with no missing data. We found that $32 \%(n=8)$ of patients had never had 25-OHD at baseline. After PDSA cycle 3, all patients had $25-\mathrm{OHD}$ checked at some point.

\section{PROBLEM}

As part of management of lytic bone lesions in patients with multiple myeloma, patients are given either Zoledronic Acid or Pamidronate Disodium at our Haematology Medical Day Unit. This project was started after an incident of a bisphosphonate induced hypocalcaemia secondary to significantly low 25-hydroxyvitamin D (25-OHD) in early September 2015. A 67 year old female with newly diagnosed multiple myeloma received Zoledronic Acid infusion. A few days later on her routine follow-up, she was found to have a low adjusted calcium of $1.74 \mathrm{mmol} / \mathrm{L}$. Of note, her adjusted calcium was normal before the infusion. Further tests revealed significantly low (25-OHD) of less than $20 \mathrm{nmol} / \mathrm{L}$, phosphate of $0.5 \mathrm{mmol} / \mathrm{L}$, parathyroid hormone (PTH) of $39.6 \mathrm{pmol} /$ L. She had no signs of hypocalcaemia. After repeat blood test, she was treated urgently with $10 \%$ of calcium gluconate infusion and was discharged home with alfacalcidol and calcium carbonate tablets with a follow-up appointment. Although this patient did not come to any significant harm, if this was left untreated, she could have potentially suffered with symptomatic hypocalcaemia and life threatening cardiac arrhythmias.

There was no formal system in place to ensure that all necessary checks (such as renal profile, bone profile, 25-OHD and dental check-ups) were performed prior to prescribing and administering bisphosphonate infusion. It relied on doctors to remind patients about having regular dental checks and ensured all necessary blood tests were requested before prescribing bisphosphonate infusion. Nursing staff administered the infusion as prescribed and may or may not aware of adverse effects of bisphosphonate infusion.

The bisphosphonate induced hypocalcaemia case described above could have been prevented with the correction of 25-OHD before initiating the infusion. The aim of this quality improvement project was (1) to avoid further bisphosphonate induced adverse effects; (2) to improve safety of bisphosphonate prescribing and administration; (3) to increase patients' awareness of needing regular dental checks. This quality improvement project was carried out at the Haematology Medical Day Unit at Epsom General Hospital, United Kingdom. The team consists of four haematologist consultants, one senior house officer (medical doctor), four nursing staff (two of which are 
specialist nurses), a ward clerk and two medical secretaries. The senior house office led the project with support and guidance from the lead consultant. We administered on average twenty bisphosphonate infusions per month at our unit.

\section{BACKGROUND}

The British Committee for Standards in Haematology (BCSH) recommends all patients with multiple myeloma should be considered for treatment with bisphosphonate infusions to reduce the risk of vertebral fractures and bone pain. ${ }^{1}$

In clinical practice, there are many drugs which require close monitoring to avoid adverse effects, these studies have used various methods to ensure accurate monitoring to improve patient's safety. ${ }^{2-4}$

According to the British National Formulary (BNF), it is necessary to correct disturbances of calcium metabolism before starting bisphosphonate infusion (2). Patients with significantly low 25-OHD are at risk of developing bisphosphonate-induced hypocalcaemia. Low vitamin D levels should be corrected by vitamin $\mathrm{D}$ supplements such colecalciferol or an active form of vitamin D (alfacalcidol). A repeat testing of 25-OHD levels should only be done in patients who have very low baseline levels and only after 3 months of treatment. Renal function and bone profile should be checked after 4 to 6 weeks of starting treatment to ensure that patients do not become hypercalcaemic due to the unmasking of primary hyperparathyroidism. ${ }^{5} \mathrm{BNF}$ advise to monitor serum electrolytes, calcium, phosphate and magnesium, and consider a dental check-up before initiating bisphosphonate due to the risk of osteonecrosis of the jaw. ${ }^{6}$

\section{BASELINE MEASUREMENT}

In order to take baseline measurements, we created a form to collect data in order to measure the scale of the problem over one month period, in October 2015. All patients who received bisphosphonate infusion within the month of October were analaysed. We would count the total number of patients who received bisphosphonate infusion, total number of patients who had dental checks, number of patients who had renal profile, bone profile and 25-OHD checked. The senior house office and nursing staff filled out the form. The senior house officer entered the collected data into a spreadsheet. These information will be collected once a month for comparison. The results were entered to a spreadsheet for analysis. There were a total of 25 patients (male, $\mathrm{n}=19$; female, $\mathrm{n}=6$ ) who attended the day unit for bisphosphonate infusion. We had to withhold the infusion from 2 of the patients. One of the patients had adjusted calcium of $1.88 \mathrm{mmol} / \mathrm{L}$ and the other patient's renal function was off his baseline. Out of 25 patients, only 9 patients $(36 \%)$ had their dental checks within the last 6 months, $2(8 \%)$ patients confirmed that they did not have dental checks within the last 6 months, and over half $(\mathrm{n}=14,56 \%)$ of the patients was labelled as "unknown". All patients $(\mathrm{n}=25)$ had urea \& electrolytes (U\&Es), renal profile and bone profiles checked. Seventeen patients $(68 \%)$ had 25-OHD checked at some point, however, there was $8(32 \%)$ patients had not had their 25-OHD checked. Out of the 17 patients who had 25-OHD checked at some point, 10 of them were due for a repeat 25-OHD.

\section{DESIGN}

We aim to have an increase in dental checks in patients from baseline of $36 \%$ to $100 \%$ within a four-month period in all patients. We also aim to have an increase in 25-OHD levels done from $68 \%$ to $100 \%$ within a fourmonth period in all patients.

After the adverse incident, we discussed the case in our Monday morning team meeting. All members of team agreed that this incident could have been prevented with various measures put in place; this includes training and education on bisphosphonate infusion and having a checklist prior to administration would be useful as an aide memoir. This also helped to establish written communication between doctors and nurses.

Based on the checklist created for baseline measurements and discussion in our Monday meeting, two checklist forms were created. One of them was for patients who were starting on bisphosphonate infusion for the first time and the other was for patients who came to our unit monthly for bisphosphonate infusion. The forms are named accordingly: (1) "Initiation: Checklist for patient prior to starting bisphosphonate infusion" and (2) "Follow-up: Checklist for patient on bisphosphonate infusion".

Both checklists are one A4 page. The initiation checklist encompassed four sections: (1) whether patient information were given (either information leaflet or verbal advice), (2) whether the patient had satisfactory dental check with a date documented on the form, (3) a table of blood test results which includes U\&Es, renal profile, bone profile and 25-OHD with three columns (dates, blood test results and comments), and (4) whether patient can be commenced on bisphosphonate infusion of either Zoledronic Acid or Pamidronate Disodium based on satisfactory checks. Under the blood results table of Section 3, we incorporated guidelines on vitamin D deficiency and the dose of colecalciferol required based on the level of deficiency. This was taken from our trust guidelines on treating vitamin D deficiency in adult patients, written based on the National Osteoporosis Society guideline on vitamin $\mathrm{D}$ and bone health. ${ }^{5}$

The follow-up checklist was very similar to the initiation checklist with three sections. It contained all sections except for Section 1 of the initiation checklist. All three sections were as follows: (1) Dental check, (2) Blood test results table and (3) Whether or not the patient should be commenced on bisphosphonate 
infusion and date of infusion. Under Section 2, there was a reminder that patients who had very low baseline 25-OHD levels will need to have a repeat testing of 25-OHD levels, 3 months after treatment. Renal function and serum calcium should be checked after 4 to 6 weeks after starting treatment with vitamin D therapy to ensure that patients do not become hypercalcaemic due to unmasking of primary hyperparathyroidism.

Training on the use of bisphosphonate infusion for patient with multiple myeloma and education on the importance of dental check and blood tests (ensuring urea \& electrolytes, renal function, bone profile and 25-OHD levels were checked) was provided by the senior house officer. The checklist was introduced in those training sessions and was emailed for all relevant members of staff which includes, consultants, haematologist specialist nurses, staff nurse, ward clerk and secretaries.

The checklist was also used as a communication tool between the doctors and nurses, especially when there was an abnormality found or treatment initiated which required further follow-up. Once all of the issues raised had been addressed, all checklists were scanned into the electronic system. This enabled all health care professionals involved in the care of the patient to have the information available to them regarding the patient's bisphosphonate infusion care. We anticipated that this process was likely to be more time consuming and may not be a favourable change as a result. However, patient safety was emphasised throughout the process to encourage participation.

\section{STRATEGY}

Interventions were modified over multiple Plan-Do-Study-Act (PDSA) improvement cycles to improve bisphosphonate infusion monitoring and patient safety. We conducted a total of three PDSA cycles. Each PDSA cycle was conducted with an interval of four weeks. Checklists were collected and entered into a database after each PDSA cycle intervention to help us identify areas that needed improvement. A weekly meeting was held (on Monday morning) once a week to discuss any issues or benefits since interventions between each cycle.

\section{PDSA Cycle 1 Intervention}

Once both initiation and follow-up forms were created and approved by the clinical lead of the haematology department, these checklists were enrolled to team members (doctors and nurse) to be used. According to the baseline measurements, there were many gaps left unfilled when to came to the dental check section. $56 \%$ $(n=14)$ of the dental check section was left blank. $32 \%$ $(n=8)$ of patients did not have 25-OHD levels checked. We discussed these results in our Monday morning meeting. All members of staff were encouraged to fill in all sections and make note of any improvements required before the next PDSA cycle. These forms were returned to the senior house office (doctor) for final review, ensuring all patients had required checks performed and any outstanding issues dealt with. This was to ensure that all patients received the same standard of care and had all necessary safety checks before receiving the infusion to avoid preventable adverse effects.

PDSA Cycle 2 Intervention

After the intervention from the last cycle, all dental checks columns were filled in with either "yes" or "no", none were left blank on the form. Only 1 patient did not have 25-OHD performed compared to 8 patients who did not have 25-OHD performed in the last month. However, there were 7 patients due to have 25-OHD levels checked. We aim to minimise this.

Teaching sessions were carried out to improve nursing staff understanding of bisphosphonate infusion and stressed the importance of checking 25-OHD and bone profile (particularly calcium levels), due to the potential of bisphosphonate induced hypocalcaemia secondary to low 25-OHD levels. We also educated nursing staff on danger of cardiac arrhythmias with dangerously low calcium levels. In addition, we also emphasised the importance of reminding patients to have initial and 6-monthly dental checks due to the risk of osteonecrosis of the jaw. As nursing staff regularly came into contact with patients, this would create an opportunity for them to educate and increase awareness amongst the patients.

Both initiation and follow-up checklists were modified and improved based on feedback from users, which includes doctors and nurses. They would like more space added for comments and annotations. There were an extra column added to the blood results section for any comments and annotations to be made of patient's blood test results. Moreover, the "comments" column could be used to record patient's baseline renal function for comparison. Furthermore, more writing space was created on the last section of the checklists to allow team members write any comments of or outstanding issues for final review. The aim was to improve communication between healthcare team of any outstanding issues and comments.

\section{PDSA Cycle 3 Intervention}

The number of patient who were due to have a 25-OHD check were down from 7 to 2 patients. All patients' dental check statuses were known. Patient and nursing staff found the reminders of getting a 6-monthly dental check were useful. They also found that the new comments columns were useful. However, there was a small proportion of patients $(n=6)$ due for a 6 -monthly dental check. In addition, we found it troublesome that we needed to go through a pile of paperwork before we can find previous records on dental checks, blood test results, vitamin D and calcium management. Therefore, we proposed that all recorded checklists were scanned to the electronic patient records system once the checklist has gone through the final review and outstanding issues were dealt with. 
The aim was to improve and extend communication to consultants clinicians who follow-up patients regularly at outpatient clinic. This allowed easy access to the documentation and information regarding patients bisphosphonate treatment. Nursing staff received further training on the management of vitamin D deficiency and bone health in myeloma patients. The management of vitamin $\mathrm{D}$ deficiency and calcium supplementation were explained to all medical doctors and nursing staff. This increased staff confidence and competency in dealing with patients with vitamin $\mathrm{D}$ deficiency.

\section{RESULTS}

After the first PDSA cycle intervention, a total of 19 patients attended the unit for bisphosphonate infusion, with only 1 patient who did not receive the infusion due to tooth extraction. Thirteen $(68 \%)$ out of 19 patients had dental checks within the last 6 months, the remaining 6 patients $(32 \%)$ did not have dental check within the last 6 months. All patients had renal function and bone profile checked prior to infusion. One patient had renal function off from baseline and the dose of the infusion was adjusted accordingly based on manufacturer's guide. All patients were found to have normal renal function test in this cycle. There were 18 out 19 patients who had 25-OHD checked at some point, however 7 of those patients were due to have a repeat 25-OHD as it was not performed within the last 3 months.

After the second PDSA cycle intervention, there was also a total of 19 patients who attended the unit for bisphosphonate infusion. All patients received bisphosphonate infusion, with 6 patients required to have their doses adjusted due to abnormal renal function. Thirteen $(68 \%)$ out of 19 patients dad dental checks within the last 6 months, remaining 6 patients (32\%) did not have dental check within the last 6 months. All patients had renal function and bone profile checked prior to infusion. Two patients had off-baseline renal function and one patient had a mildly abnormal adjusted calcium level of $2.06 \mathrm{mmol} / \mathrm{L}$. Eighteen out of 19 patients had 25-OHD performed at some point with only 2 patients due for a recheck. Only 1 patient had not had 25-OHD performed at any point; 25-OHD was done on the same day.

After the third PDSA cycle intervention, there was an improvement of up to $69 \%(n=11)$ of patients had dental checks within the last 6 months. Similarly to the previous PDSA cycles, all patients had renal function and bone profile performed prior to infusion. By this stage, all patients $(n=16)$ had 25-OHD performed at some point. Out of the 16 patients, only 4 patients were due for a repeat 25-OHD. All patients received bisphosphonate infusion, with two patients having had doses adjusted according to their renal function.

There was an overall improvement in ensuring safety checks were done prior to administration of bisphosphonate infusion compared to baseline measurements. See Table 1 and Figure 1 for comparison of each PDSA cycle to the baseline measurement.

\section{LESSONS AND LIMITATIONS}

A main limitation of this project was the small sample size and that we have only collected data over a fourmonths period. The improvement shown in this study could be due to chance and as there was no statistical testing. Ideally, sample size should be larger at a longer period. This was not possible due to the small number of patients that receive bisphosphonate infusion at our unit. We had an average of 20 patients each month who received this infusion. The baseline measurement was limited as the relevant data points were not being recorded prior to this study. We attempted to establish a baseline for this project by collecting existing data using a data collection sheet. The data collection sheet itself may have prompted members of staff to perform more checks that otherwise would not have happened. One of the challenges was getting team members to participate in the process earlier on the study. The process of filling out a checklist may have been perceived as time consuming. If a similar project were initiated, we would recommend early staff training and education to justify the change in practice.

The checklist provides guidance especially for nonspecialist doctors who are new to the unit or unfamiliar with prescribing bisphosphonate infusions. This provides safer practice and avoid preventable adverse effects related to bisphosphonate infusion. To ensure the sustainability of this project, we have included the guidance on prescribing bisphosphonate infusion and management of vitamin D deficiency in the Junior Doctors Haematology Handbook for the next senior house officer (doctor) when he or she takes over. All consultants and nursing staff are actively involved in the

Table 1 Summary of results of dental check and 25-OHD at baseline, post PDSA cycle 1, 2 and 3.

\begin{tabular}{llllllll}
\hline & \multicolumn{2}{l}{ Dental Check (within last $\mathbf{6}$ months), \% (n) } & & \multicolumn{2}{l}{ 25-OHD, \% (n) } \\
\cline { 2 - 4 } \cline { 6 - 8 } & Yes & No & Unknown & & Due & Not Due & Not Done \\
\hline Baseline (Oct) & $36.0(9)$ & $8.0(2)$ & $56.0(14)$ & & $40.0(10)$ & $28.0(7)$ & $32.0(8)$ \\
PDSA-1 (Nov) & $68.4(13)$ & $31.6(6)$ & $0.0(0)$ & $36.8(7)$ & $57.9(11)$ & $5.3(1)$ \\
PDSA-2 (Dec) & $68.4(13)$ & $31.6(6)$ & $0.0(0)$ & $10.5(2)$ & $84.2(16)$ & $5.3(1)$ \\
PDSA-3 (Jan) & $68.8(11)$ & $25.0(4)$ & $6.3(1)$ & $25.0(4)$ & $75.0(12)$ & $0.0(0)$ \\
\hline
\end{tabular}


Figure 1 Bar charts show percentages of patients' dental check and 25-OHD check status.
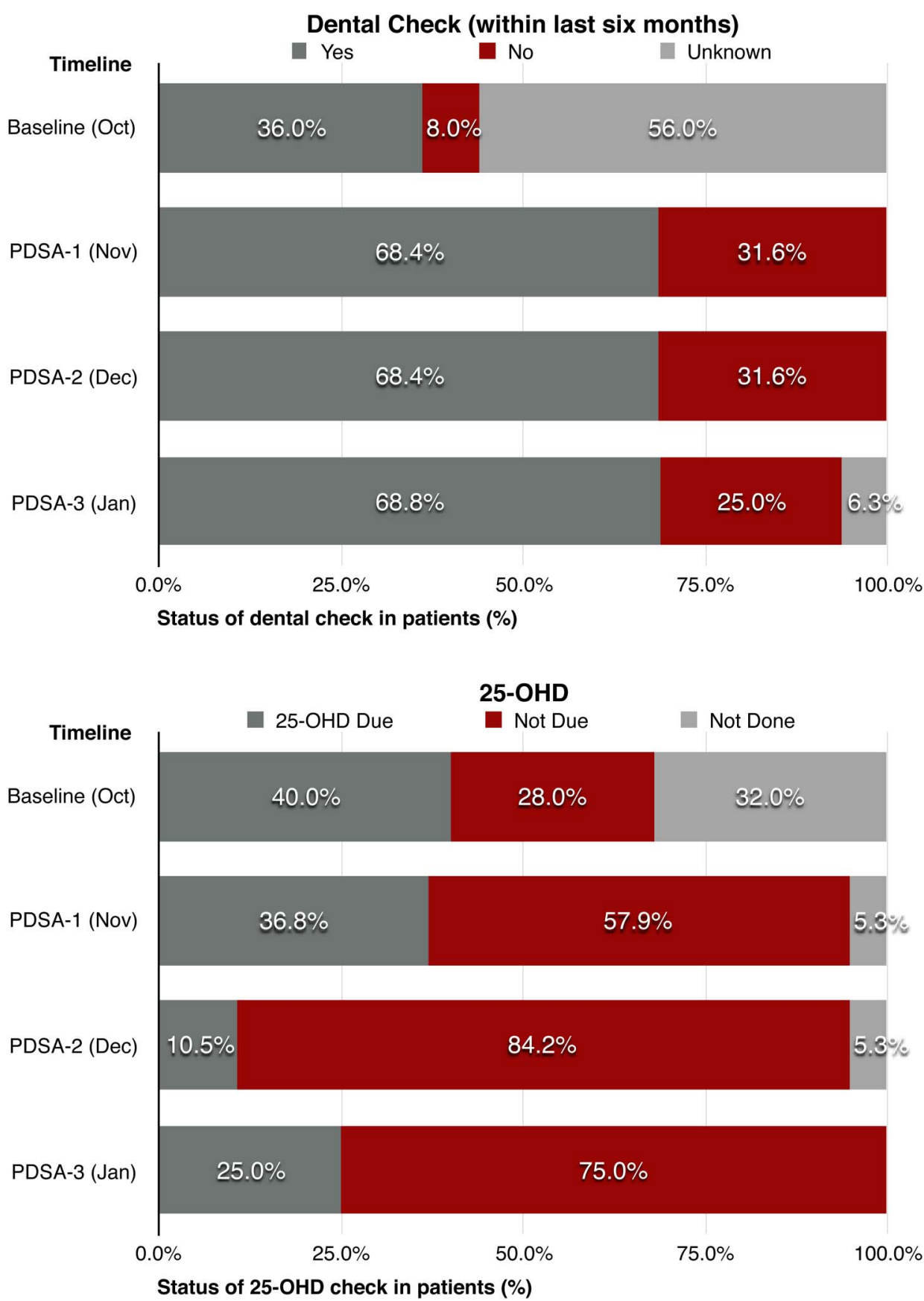

current process and it is a requirement that all this checklist form is filled out before administration of bisphosphonate infusion.

Although more rigorous recordings of 25-OHD and other blood tests may have led to more tests being requested, this is in line with best practice guidelines for bisphosphonate infusion. The anticipated reduction of adverse incidents should also be considered in the overall cost effectiveness of this process. Further study in this area would be required to confirm this.

\section{CONCLUSION}

Our project aimed to avoid preventable bisphosphonate induced adverse effects, to improve safety of prescribing and administrating bisphosphonate infusion, and to increase patients' awareness of needing regular dental checks. Since the intervention, there have been no further bisphosphonate-related adverse events. The collation of data relevant to bisphosphonate infusion prescription on the checklist has led to more informed prescribing and administration of medication. Any abnormalities found from the checklist were dealt with accordingly and infusions were withheld on three occasions over the period of this study to avoid potential adverse effect. In regards to dental checks, the status of over half of our patients were unknown at baseline. After intervention, more than two thirds of patients had checks within the last 6 months and we were more 
confident that patients had been advised to book checkups and instruction was being given by nursing staff. Furthermore, this change has also gained interest from another unit within the Trust and they are reviewing their bisphosphonate infusion monitoring process and would like to incorporate these ideas into their monitoring process.

In conclusion, the initiation and follow-up checklists represent a standardised method and could be adapted to other similar situations to ensure all safety precautions are applied. This project was initiated after an adverse incident; although this project may appear to meet its original aims and objectives, further studies on the cost effectiveness of this intervention is recommended.

Acknowledgements Special thanks Tracy Reeman, Sadie Burrage, Enkelejda Qalliaj, Laura Moran, Louisa Skyes and Sarah Spencer for all their efforts in this project.

Declaration of interests Nothing to declare.

Ethical approval Ethical approval was not required since it was an improvement project to an existing process. It was not a study on human subjects.
Open Access This is an open-access article distributed under the terms of the Creative Commons Attribution Non-commercial License, which permits use, distribution, and reproduction in any medium, provided the original work is properly cited, the use is non commercial and is otherwise in compliance with the license. See:

- http://creativecommons.org/licenses/by-nc/2.0/

- http://creativecommons.org/licenses/by-nc/2.0/legalcode

\section{REFERENCES}

1. Bird J, Owen R, DSa S, et al. Guidelines for the diagnosis and management of multiple myeloma 2014. British Committee for Standards in Haematology 2014.

2. Karlsson T. A project to improve the management of patients on warfarin in a primary care setting through the introduction of a POC analysis. BMJ Qual Improv Rep 2016;5:u211003.w4421. doi:10.1136/bmjquality.u211003.w4421

3. Williamson A, Bradley A, Khan K. Using a simple handover to improve the timing of gentamicin levels. BMJ Qual Improv Rep 2015;4:u207727.w3081. doi:10.1136/bmjquality.u207727.w3081

4. Qureshi DI, Habayeb MH, Grundy DC. Improving the correct prescription and dosage of gentamicin. BMJ Qual Improv Rep 2012;1:u134.w317. doi:10.1136/bmjquality.u134.w317

5. Francis R, Aspray $\mathrm{T}$, Fraser W, et al. Vitamin D and bone health - a practical guideline for patient management. National Osteoporosis Society 2013.

6. Joint Formulary Committee. British National Formulary (BNF) 71 2016 ed. Pharmaceutical Press 2016. 